\title{
Crowberry Inhibits Cell Proliferation and Migration Through a Molecular Mechanism that Includes Inhibition of DEK and Akt Signaling in Cholangiocarcinoma
}

\author{
Xue Wang \\ Yanbian University \\ Ludan Zhang \\ Yanbian University \\ Xin Zhang \\ Yanbian University \\ Xuebing Zhou \\ Yanbian University \\ Chunyu Yang \\ Yanbian University \\ Yingshi Piao \\ Yanbian University \\ Jinhua Zhao \\ Yanbian University \\ Lili Jin \\ Yanbian University \\ Guihua Jin \\ Yanbian University \\ Renbo An \\ Yanbian University \\ xiangshan ren ( $\nabla$ renxsh@ybu.edu.cn ) \\ Yanbian University
}

\section{Research Article}

Keywords: Cholangiocarcinoma, Empetrum nigrum var. japonicum, Proliferation, Migration, DEK

Posted Date: February 1st, 2022

DOI: https://doi.org/10.21203/rs.3.rs-1265629/v1 
License: (c) (i) This work is licensed under a Creative Commons Attribution 4.0 International License. Read Full License 


\section{Abstract}

Background: Cholangiocarcinoma (CCA) is a rare biliary adenocarcinoma related to poor clinical prognosis. Crowberry is an herbal medicine used to control inflammatory diseases and reestablish antioxidant enzyme activity. Although crowberry shows significant therapeutic efficacy in various tumors and diseases, its anticancer effects and specific molecular mechanisms in CCA are poorly understood.

Aim of the study: This study was conducted to characterize crowberry effects on CCA cells behavior.

Materials and methods: The chemical profiles of crowberry extract was qualitatively analyzed by highperformance liquid chromatography (HPLC) and HPLC--tandem mass spectrometry. MTT, colony formation and EdU assays were performed to measure cell proliferation. The effect of crowberry treatment on CCA cell migration was assessed by wound healing and migration assays. Moreover, Hoechst staining assay and flow cytometry were performed to assess the cell apoptosis rate. Western blotting was used to assess the protein expression levels of key factors associated with apoptosis, the Akt signaling pathway, and the epithelial-mesenchymal transition. A xenograft model was established and immunohistochemical and H\&E staining was performed to assess crowberry antitumor effects in vivo.

Results: Crowberry clearly inhibited CCA cells proliferation and migration in a dose-dependent manner and induced apoptosis in vitro. Crowberry inactivated the PI3K/Akt signaling pathway by regulating DEK in vitro and significantly inhibited tumor growth by downregulating the $D E K$ expression in xenograft models.

Conclusion: Crowberry inhibits CCA cells proliferation and migration through a molecular mechanism that includes inhibition of $D E K$ and Akt signaling pathway inhibition in vitro and in vivo.

\section{Introduction}

Cholangiocarcinoma (CCA) is a malignant cancer that originates from the bile duct epithelial cells and is associated with poor outcomes. Even after major clinical interventions, such as surgery, radiation therapy, and chemotherapy, the survival rate is not significantly improved and remains low with only $15 \%$ surviving to five years after treatment ${ }^{[1-2]}$. Because of poor clinical outcomes, more effective therapeutic options that induce low toxicity are urgently required.

Empetrum nigrum var. japonicum Siebold \& Zucc. ex K.Koch, also known as crowberry, is a small class of creeping evergreen shrubs and an undomesticated bacca that has considerable herbal potential ${ }^{[3]}$. It exhibits both anti-inflammatory and anticancer functions. For example, it has been used to maintain youth and treat inflammatory diseases, such as cystitis and urethritis ${ }^{[4-5]}$. Moreover, ultraviolet $B$ radiation-induced cell damage can be repaired by crowberry extract treatment ${ }^{[6]}$. In addition, recent studies have shown that crowberry contains important bioactive compounds ${ }^{[7-8]}$, and several of these components, including (2S)-5'-hydroxy-7,3',4'- trimethoxyflavanone, 2',4'-dihydroxy-3'-methoxychalcone, 4'- 
hydroxy-7-methoxyflavanone and 2',4'-dihydroxychalcone, have shown strong anticancer effects on P-388 lymphoid leukemia and HT-29 colon cancer cell ${ }^{[9]}$. In addition, crowberry is recorded in Hulunbeier Mongolian Medicine Resources, because its branches and leaves can be used as supplements to treat the spleen and stomach, particularly treat indigestion, and the fruit nourishes the liver; People often use crowberry as a medicine for the prevention and treatment of alcoholic liver disease, and it reportedly has a satisfactory effect as a Chinese folk ${ }^{[10]}$. Although significant therapeutic effects of crowberry on various diseases and tumors have been reported, the specific molecular mechanisms of crowberry action in CCA are not well understood.

$D E K$ is an oncoprotein on human chromosome $6 \mathrm{p} 22.3$ of. Acetylation reduces the affinity of $D E K$ for DNA and inhibits deacetylase resulting in the accumulation of particle clusters DEK (IGC) in chromatin and RNA processing factors comprising subnuclear structures. Subsequently, through the abovementioned complex posttranslational modifications, the following processes are realized: RNA transcription, RNA splicing, DNA replication and DNA repair ${ }^{[11]}$. DEK has been proven to promote the formation and development of solid tumors: for instance, $D E K$ is highly expressed in breast cancer and is associated with a malignant phenotype and progression ${ }^{[12]}$. High DEK expression can promote the epithelial-tomesenchymal transition (EMT) process of esophageal squamous carcinoma cells, accelerate lymph node metastasis, and significantly reduce the overall survival rate of patients ${ }^{[13]}$. High expression of DEK in pancreatic cancer has been found to promote lymph node metastasis and to be related to poor prognosis ${ }^{[14]}$. Additionally, the overall survival rate of liver cancer patients with high $D E K$ expression is significantly reduced ${ }^{[15]}$. However, DEK expression is rarely reported in CCA. The Akt/mTOR pathway is essential for the proliferative and migratory potential of cells. Various diseases, such as inflammation and cancer, can develop when signaling through the Akt/mTOR pathway is inefficient ${ }^{[16-17]}$. Natural plants that can attenuate the liver fibrosis by regulating the phosphorylation of components in the Akt/mTOR pathway have attracted attention ${ }^{[18]}$. Actually, the GeneMANIA database indicates that there is a significant connection between $D E K$ and the Akt/mTOR signaling pathway. DEK participates in tumor cell proliferation, apoptosis invasion and differentiation by regulating several signaling pathways, such as Akt signaling pathways, thereby promoting tumor cell growth and infiltration ${ }^{[19-20]}$. These studies indicate that $D E K$ can potentially be used as a target for the development of antitumor drugs.

The main purpose of this study was to characterize the inhibitory effect of crowberry on the HuCCT1 and QBC939 CCA cell lines and elucidate the molecular mechanism of its action. Taken together, this study provides new ideas and a theoretical basis for the clinical treatment of CCA.

\section{Materials And Methods}

\section{Plant materials and extraction}

Crowberry was provided by the Key Laboratory of Natural Medicines of the Changbai Mountain ( Yanbian University ), Ministry of Education. The whole plant of crowberry was purchased in March 2018 at the 
Gen He Crude drug market in Hei Long Jiang Province, China. The voucher specimen (No. YBU-1020) was identified by Professor Hui-zi Lv, Botanical garden, Yanbian University, and stored in the Herbarium of the College of Pharmacy at Yanbian University (China). The air-dried and powdered whole plant of crowberry $(6.0 \mathrm{~kg})$ were extracted with $\mathrm{MeOH}(25 \mathrm{~L}$, reflux, $5 \mathrm{~h} \times 3)$, followed by removal of the solvent under reduced pressure to yield a dried $\mathrm{MeOH}$ extract (1.5 kg, yield 25\%). The dried $\mathrm{MeOH}$ extract was dissolved in dimethyl sulfoxide (DMSO) to a density of $300 \mathrm{mg} / \mathrm{ml}$ and deposited at $-20^{\circ} \mathrm{C}$ until analysis.

\section{High-performance liquid chromatography and HPLC- tandem mass spectrometry (MS/MS)}

Chromatographic experiments were performed using an HPLC instrument equipped with a 5260 autosampler and 5430 diode-assay UV/Vis detector (DAD) (HITACHI Chromaster, Japan) and mass spectrometry (Agilent, 6460, USA). For all experiments, a LaChrom ODS C-18 column (250 mmL ×4.6 mml.D., $5 \mu \mathrm{m}$, HITACHI, Japan) was used as the stationary phase, and the syringe amount was $5 \mu \mathrm{L}$. The mobile phase was composed of $0.5 \%$ phosphoric acid solution (A) and methanol (D), and the system was equilibrated for 20 min with the beginning conditions. The following gradient program has been applied to methanol (D): linear gradient from $58 \%$ D to $60 \%$ D for 20 minutes, $60 \%$ D increasing to $62 \%$ D in 55 min, and then $62 \%$ D increasing to $100 \%$ D for $20 \mathrm{~min}$. The column was washed with $100 \% \mathrm{D}$ for $10 \mathrm{~min}$, and the flow rate was $0.7 \mathrm{~mL} / \mathrm{min}$. UV absorption at $250 \mathrm{~nm}$ was measured to define the analyses (Fig. 1). Then obtain the corresponding chromatographic peaks for 2'-methoxy-4'-hydroxydihydrochalcone(1), 2',4'-dihydroxy-dihydrochalcone(2), and 2',4'-dihydroxy-chalcone (3).

\section{Cell lines and cell culture}

CCA cell lines, RBE and QBC939, were purchased from the Cell Bank of Chinese Academy of Sciences (Shanghai, China). The human cholangiocarcinoma cell lines, HuCCT1 and TFK1, were gifted by Kanazawa University in Japan. Normal human bile duct epithelial cell HIBEpic was obtained from American Type Culture Collection (ATCC, Manassas, VA, USA). Cell lines above were grown in RPMI-1640 medium (GIBCO, Gaithersburg, MD). Cells were supplemented 1\% penicillin/streptomycin and $10 \%$ fetal bovine serum (GIBCO, Gaithersburg, MD). Cells were incubated at $37^{\circ} \mathrm{C}$ with a $5 \% \mathrm{CO} 2$ atmosphere.

\section{MTT assay}

HuCCT1 and QBC939 cells were seeded 5000 cells/well in a 96-well plate, and treated with crowberry at various concentrations for $24,48,72 \mathrm{~h}$. Correspondingly, silenced and overexpressed DEK cells were cultured for 48 hours. Briefly, the value of absorbance was measured at a full-wavelength spectrophtotmeter (TECAN-infinite M200 pro, Switzerland).

\section{Colony formation assay}

Cells were seeded in six-well plates and then cultured with crowberry for 2 weeks. The visible colonies were fixed with $4 \%$ paraformaldehyde and stained with $1 \%$ crystal violet (Solarbio, China). ImageJ 
software was used to analysze the data.

\section{EdU assay}

Cells were seeded at 5000 cells/well in 96-well plates. After treatment, these cells were cultured in complete medium containing $50 \mu \mathrm{M}$ EdU (RiboBio, China) for 2 hours at $37^{\circ} \mathrm{C}$ followed by washing twice with phosphate-buffered saline (PBS). The cells were fixed and permeabilized and then, EdU was detected following the EdU assay kit manufacturer's instructions. Finally, cell immunostaining was observed and images were acquired with a fluorescence microscope (Olympus, Japan).

\section{Hoechst33342 staining}

After treating with the indicated crowberry for $48 \mathrm{~h}$, cells were fixed with $4 \%$ formaldehydefor, and stained for $15 \mathrm{~min}$ with $1 \mu \mathrm{g} / \mathrm{ml}$ Hoechst33342 in the dark. The cells were then washed twice with PBS. A fluorescence microscope was used to image the cells.

\section{Flow cytometry}

Cells treated with different concentrations of crowberry after $48 \mathrm{~h}$. The cells were washed with PBS and re-suspended in binding buffer. Cells were stained in the dark with FITC-annexin V and PI (BD Biosciences, USA) for 15 min. BD Accuri C6 (BD Biosciences) was used to detect the samples.

\section{Wound healing assay}

Cells were cultured in 6-well plate to $80 \%$ confluence. The wounds were created by $200 \mu$ micropipette tip and replaced with different doses of crowberry. Cell migration was observed at 0,12 and 48 hours using microscopy (Olympus, Japan). Silenced or overexpressed DEK cells were observed at 12 hours. Image J software was used to analyze the results.

\section{Cell migration assay}

Cells were seeded in the upper chambers of Transwell inserts (Costar, Corning Incorporated, USA), at a concentration of $5 \times 10^{4}$ cells/well. Then the cells were incubated with medium containing $1 \%$ fetal bovine serum (FBS) for 4 hours. Medium containing $10 \%$ or $20 \%$ FBS was added to the lower chamber. Specifically, QBC939 cells were cultured for 72 hours, and HuCCT1 cells were cultured for 24 hours. Cells were fixed with $4 \%$ paraformaldehyde and then stained with $1 \%$ crystal violet. Cells were photographed with an Olympus BX53 microscope.

\section{Transfection}

DEK small interfering RNA (siRNA) reagent was purchased from RiboBio (China). The effective sequence of the DEK gene used here was (5'-TGTCCTCATTAAAGAAGAA-3). The cells were incubated with $50 \mathrm{nM}$ siRNAs containing Lipofectamine 3000 (Invitrogen, Carlsbad, CA) for 48 hours. An lentiviral vector overexpressing DEK (DEK (Human, NM_003472)) was purchased from Beijing Syngentech Co., Ltd, China, which serial number is pHS-AVC-0466. HuCCT1 cells were transfected with this vectorin a 6-well plate, 
and $2 \mathrm{ml}$ of medium supplemented with $2 \mu \mathrm{l}$ of polybrene and $2 \mu \mathrm{l}$ of lentivirus was added for transfection. After 8 hours on incubation, the medium was replaced with fresh medium, and the cells were incubated for another 72 hours. The $D E K$-transduced cells were incubated with $5 \mu \mathrm{g} / \mathrm{ml}$ puromycin in the medium.

\section{Western blotting}

After the cells were treated with crowberry for 48 hours, total proteins $(40 \mu \mathrm{g})$ were quantified with BCA protein assay (Beyotime, Shanghai, China), electrophoresed through SDS-PAGE, and transferred to PVDF membrane (Millipore, Eschbom, Germany). The membrane was incubated with primary antibodies Bax (1:1000), Bcl-2 (1:1000), Cleaved-Caspase3 (1:1000), Cleaved-Caspase9 (1:1000), Cleaved-PARP (1:1000), Cytoc (1:1000), p-Akt (1:1000), Akt (1:1000), p-S6 (1:1000), S6 (1:1000), p-4EBP1 (1:1000), 4EBP1 (1:1000), E-cadherin (1:1000), Vimentin (1:1000), and Snail (1:1000), which were obtained from Cell Signaling Technology, USA, and DEK (1:1000, BD, USA), actin (1:3000, Abcam, USA) at $4{ }^{\circ} \mathrm{C}$ overnight. Secondary antibody (1:3000, Beyotime, China) was added at RT for $1 \mathrm{~h}$. Enzymatic signals were visualized with a ChemiDoc Touch Imaging System (Bio-Rad, USA), and statistics were performed with ImageJ software.

\section{Xenograft model}

The study was approved by the Ethics Committee of Yanbian University. BALB/C female nude mice (4 weeks old) were obtained from Changzhou Cavens Laboratory Animal Co., Ltd., China. Each mouse was injected with $2 \times 10^{6} / 100 \mu \mathrm{QBC} 939$ cells subcutaneously into the rigtht armpit. One week after inoculation, 16 mice were randomly assigned into 2 groups (8 mice in each group). Every day, mouse body weight was recorded, and tumors were measured with a Vernier caliper; the tumor volume was ascertained following the formula: tumor volume $=0.5 \times\left(\right.$ length $\times$ width $\left.^{2}\right)$. The treatment group was treated every day with crowberry $(50 \mathrm{mg} / \mathrm{kg}$ ) diluted with $0.1 \%$ methylcellulose for intragastric administration (ig), and the same volume of methylcellulose was used to treat the control group mice. On day 30 , the mice were anesthetized with $1 \%$ pentobarbital sodium and then sacrificed. Mouse livers and tumors were fixed in $4 \%$ paraformaldehyde for immunohistochemical and hematoxylin and eosin (H\&E). This experiment was completed in a specific-pathogen-free (SPF) animal laboratory in the Yanbian University Animal Experiment Center. The experiment was approved by the Animal Protection Committee of Yanbian University (SCXK (JI)2017-0003). the research was conducted in accordance with internationally accepted principles (European community guidelines) for laboratory animal use and care.

\section{Immunohistochemistry staining (IHC)}

The fixed mouse tumors were embedded in paraffin and then sliced into $4 \mu \mathrm{m}$ thin slices. Antigen was retrieved in $100^{\circ} \mathrm{C}$ citrate buffer for $10 \mathrm{~min}$. After endogenous peroxidase was blocked with $1 \%$ hydrogen peroxide for 10 minutes, the Ki67 antibody (1:100, Affinity Biosciences, rabbit monoclonal, USA) and DEK antibody (1:100, protheintech, Mouse monoclona, USA) were incubated at $4{ }^{\circ} \mathrm{C}$ overnight. After incubation with secondary antibody, the section was observed with diaminobenzidine (DAB, Zhongshan 
Golden Bridge PV- 9000, Beijing, China), and the nucleus was stained with hematoxylin. Five fields of 400x view were selected to count the number of Ki67-positive cells.

\section{Statistical analysis}

Statistical analyses were primarily conducted by GraphPad Prism 8.0 software (GraphPad, San Diego, CA) and SPSS 20.0 software (SPSS, Chicago, IL). One-way ANOVA was used for analysis multiple comparisons. Two group comparisons were performed using T-test. $P$-Values $<0.05$ were considered as significant.

\section{Results}

\section{Bioactive compounds in crowberry extract}

The existence of flavonoid monomers of the chemical compounds in the methanol extract of crowberry extract has been shown using HPLC-MS/MS analysis. Fig. 1 shows the HPLC chromatograms recorded at $250 \mathrm{~nm}$, the main dominant compound as 2',4'-dihydroxy-chalcone. The contents of 2'-methoxy-4'hydroxy-dihydrochalcone (1), 2',4'-dihydroxy-dihydrochalcone (2), and 2',4'-dihydroxy-chalcone (3) were $0.71 \%, 1.15 \%$ and $1.35 \%$, respectively.

\section{Crowberry suppresses the proliferation and colony formation of CCA cell lines}

Polyphenolic compounds are a major group of secondary metabolites that have the anticancer property. To assess the inhibitory effects of crowberry on CCA cells, the 3-(4,5-dimethylthiazol-2-yl)-2,5 diphenyltetrazolium bromide (MTT) assay was used to detect cell viability. HuCCT1 and QBC939 cells were treated with different concentrations of crowberry. The result showed that crowberry profoundly inhibited the cell viability of HuCCT1 and QBC939 cells in a dose- and time-dependent manner compared with control group cells. Then the half-inhibition rate (IC50) of HuCCT1 $(87.5 \mu \mathrm{g} / \mathrm{ml})$ and QBC939 (144.1 $\mu \mathrm{g} / \mathrm{ml}$ ) were calculated. Accordingly, the concentrations of crowberry selected for further experiments were 40,80 , and $160 \mu \mathrm{g} / \mathrm{ml}$ for HuCCT1 and 100,150 , and $225 \mu \mathrm{g} / \mathrm{ml}$ for QBC939 cells $(P<0.05$ or $P<$ 0.01 ; Fig. $2 A$ ). Then, the proliferative ability was determined using colony formation and 5-ethynyl-2'deoxyuridine (EdU) assays, and the results showed that crowberry significantly reduced the proliferation of HuCCT1 and QBC939 cells in a dose-dependent manner compared with control group cells $(P<0.01$; Fig. 2B-2C). In conclusion, crowberry inhibits the proliferation of CCA cells.

\section{Crowberry induces the apoptosis of CCA cells}

To determine whether crowberry has an apoptotic effect on CCA cell lines, we performed Hoechst 33342 staining,which revealed an obvious induction of apoptosis in HuCCT1 and QBC939 cells following crowberry treatment (Fig. 3A). Similarly, the results of Annexin-V and PI staining showed that the number of apoptotic cells increased significantly after treatment with crowberry compared to the control cells $(P<$ 
0.05 or $P<0.01$; Fig. 3B). Moreover, crowberry significantly increased the level of pro-apoptotic proteins compared with control group cells, including cleaved caspase-9, cleaved caspase-3, cleaved-PARP, and Bax. Similarly, crowberry treatment also increased the level of key mitochondrial apoptotic pathway factor Cyto $\mathrm{C}$, whereas the expression of the anti-apoptotic gene $\mathrm{Bcl} 2$ was clearly reduced (Fig. $3 \mathrm{C}$ ). These consequences demonstrate that crowberry induces the apoptosis of CCA cells.

\section{Crowberry suppresses the migration and EMT of CCA cells}

Given the close connection between EMT and tumor migration ${ }^{[21]}$, we examined the effects of crowberry on EMT in CCA cells using wound healing, transwell, and western blotting assays. Crowberry significantly reduced the horizontal and vertical migration of CCA cells in vitro contrast to control group cells $(P<0.01$; Fig. 4A-4B), as defined by wound healing and transwell assays. Furthermore, crowberry increased the expression of E-cadherin, whereas it inhibited the level of Vimentin and Snail (Fig. 4C). To prove that the suppressed migration was not from suppression of cell growth or cell death caused by the relatively high concentration, HuCCT1 and QBC939 cells were treated with lower concentration of crowberry, followed by MTT assay and wound healing assay. The results showed that when the concentrations were $20 \mu \mathrm{g} / \mathrm{ml}$ and $50 \mu \mathrm{g} / \mathrm{ml}$, the cell viability of HuCCT1 and QBC939 cells was not affected $(P<0.01$; supplementary Fig.A), but the horizontal migration ability was significantly inhibited $(P<0.01$; supplementary Fig.B). These results indicate that crowberry prevents EMT and migration in CCA cells.

\section{Crowberry inhibits the Akt/mTOR signaling pathway}

We next examined the level of key mediators of the Akt/mTOR signaling pathway. Crowberry treatment obviously decreased the phosphorylated level of Akt, S6, and 4EBP1 compared with control group cells (Fig. 5), suggesting that crowberry attenuates the proliferative potential of CCA cell by inhibiting Akt/mTOR pathway.

\section{Crowberry suppresses cell proliferation and migration by regulating DEK}

$D E K$ plays important roles in the proliferation, migration, and metastasis of tumor cells ${ }^{[22-23]}$. Due to understand the molecular mechanism of crowberry action in detail, we detected DEK expression between HIBEpic and CCA cells. Fig. 6A demonstrated that DEK significantly upregulates in CCA cells compared with HIBEpic cells. Additionally, the levels of $D E K$ significantly reduced after being treated with crowberry (Fig. 6B). We then silenced and overexpressed DEK in HuCCT1 cells to demonstrate the potential oncogenic role of $D E K$ in CCA cells (Fig. 6C). As a result, a significant reduction in cell proliferation was observed in siDEK cells and the result in overexpression DEK cells was reversed (Fig. 6D-E). Moreover, lateral and longitudinal migration abilities were significantly weakened compared with control group. In contrast, the migration abilities of HuCCT1 cells were significantly enhanced after overexpression of DEK (Fig. 6F). Above all, these data indicated that DEK is overexpressed in CCA cell lines, and cowberry suppression of DEK expression may contribute to its roles in CCA inhibition. 


\section{Crowberry targets DEK and AKt/mTOR signaling pathway to inhibit EMT progression in CCA cells}

Furthermore, $D E K$ silencing downregulated $\mathrm{p}$-Akt, $\mathrm{p}-4 \mathrm{EBP} 1$, and $\mathrm{p}-\mathrm{S} 6$ expression compared with control group cells, and overexpression cells were reversed $(P<0.05$ or $P<0.01$; Fig. 7A). Additionally, E-cadherin expression was upregulated after $D E K$ silencing, whereas the Vimentin and Snail expression were downregulated compared with control group cells. As we speculated, the results were reversed in HuCCT1 cells overexpression DEK (Fig. 7B). It implied that DEK exerts oncogenic functions via Akt signaling pathway modulationin CCA cells.

\section{Crowberry inhibits CCA progression in vivo}

To further convince the vitro observations, xenograft model was performed. The general view of nude mice and dissected tumors are shown in Fig. 8A. Unfortunately, one mouse of $50 \mathrm{mg} / \mathrm{kg}$ group died during ig. Another mice in treatment group has no tumor, and was not included in the statistics. Compared with the control group, there was no significant change in the weight of the nude mice (Fig. 8B). Fig. 8C-D showed that the $50 \mathrm{mg} / \mathrm{kg}$ group tumors exhibited a significant reduction in volume and weight $(P<0.01)$ compared with control group. To evaluate the toxic effect of crowberry, $\mathrm{H} \& \mathrm{E}$ staining was performed. The results showed that the liver fiber was intact without inflammatory cell infiltration (Fig. 8E), suggesting that there was no damage of crowberry at a dose of $50 \mathrm{mg} / \mathrm{kg}$. Further, the IHC staining was performed, indicating that Ki67 and DEK were significantly decreased in the xenograft tumor tissues compared with the control group. The Ki67 indexes were $83.2 \%, 7.4 \%$ respectively in control and $50 \mathrm{mg} / \mathrm{kg}$ group (Fig. 8F-J). Thus, crowberry inhibits CCA proliferation via DEK in vivo.

\section{Discussion}

CCA is a malignant tumor of the digestive tract with a high mortality rate ${ }^{[24-25]}$. Due to immunomodulatory, antibacterial, and even antitumor effects, natural plant caused a widespread concern $^{[26]}$. As previously mentioned, crowberry shows significant potential efficacy on the treatment of various diseases, but its antitumor effects on CCA have not been fully elucidated. In this study, we found that crowberry extracts significantly suppressed the cell proliferation and metastasis of HuCCT1 and QBC939 cells. Particularly, it showed that the $50 \mathrm{mg} / \mathrm{kg}$ group tumors exhibited a significant reduction in volume and weight in vivo. The HPLC-MS/MS analysis of crowberry showed obvious peaks for 2'methoxy-4'-hydroxy-dihydrochalcone (1), 2',4'-dihydroxy-dihydrochalcone (2), and 2',4'-dihydroxy-chalcone (3) contents compared with other plant Ixeridium gracile it is higher ${ }^{[27]}$. Previous studies indicated that 2',4'-dihydroxychalcone suppresses PC-3 human prostate cancer cell growth by inducing apoptosis ${ }^{[28]}$.

Apoptosis induction is one of the key mechanisms of anticancer medicine ${ }^{[29]}$. Many studies have shown that prohibiting the expression and/or function of Bax can resist cytochrome $\mathrm{C}$ (Cyt $\mathrm{C}$ ) released by mitochondria, prohitbit the reducing mitochondrial membrane potential, and guarantee cells against 
apoptosis $^{[30]}$. Consistent with these results, we found that crowberry reduced the expression of the prosurvival protein $\mathrm{Bcl}-2$ but elevated the expression of the pro-apoptotic protein Bax and key mitochondrial apoptotic pathway factor Cyto C. A previous study showed that Cyto C indirectly triggered initiator caspase-9 that is primarily responsible for the beginning of the apoptotic pathway and executioner caspase- 3 that is responsible for the definite cleavage of cellular components leading to apoptotic cell death $^{[31]}$. In general, PARP is considered an indicator of caspase 3 activation. In our study, the protein expressions of cleaved-caspase 9 , cleaved-caspase 3 , and cleaved-PARP were increased in crowberrytreated CCA cells, further promoting the apoptosis of CCA cells. It has been reported that, in HCT116 cells, 6-gingerol induced apoptosis by upregulating the protein expression of caspase3, PARP1, and Bax and downregulating $\mathrm{Bcl}-2^{[32]}$, further validates our point of view. Overall, crowberry induces apoptosis in CCA cells.

$D E K$ is an oncogene that promotes proliferation, EMT, and metastasis in various cancers ${ }^{[33-34]}$. Previous studies have shown that $D E K$ is highly expressed in CCA and that inhibiting DEK gene expression can delay the development of $\mathrm{CCA}^{[35]}$. We found that $D E K$ is highly expressed in CCA by using the UALCAN database. In the present study, we found that DEK protein levels in CCA cell lines (HuCCT1, QBC939, and RBE) were significantly higher than in the normal human biliary cell line HIBEpic. These results are consistent with UCLAN database analysis. In our study, crowberry significantly inhibited the expression of $D E K$ in vivo and vitro. To survey the function of DEK in CCA tumorigenesis, we silenced DEK expression. Consequences demonstrated that in vitro CCA cell proliferation and migration were inhibited, and as we expected, cell proliferation and migration were enhanced when $D E K$ was overexpressed, suggesting an oncogenic role for $D E K$ in CCA. To verify that cowberry-modulated cell proliferation is related to $D E K$ induced apoptosis, we considered our previous research results, which showed no change in the CCA cell apoptosis after $D E K$ was silenced. We speculated that cowberry-modulated cell death is not related to apoptosis induction, but may be related to Akt signaling pathway inactivation (siDEK data are not shown). Yang et al. had previously discovered that $D E K$ overpresstion exerted a significant promoting effect on breast cancer cell proliferation, invasion, and migration, and that high DEK expression was positively correlated with lymph node metastasis and the Ki67 index, which indicated poor prognosis ${ }^{[12]}$. Further, the xenograft model confirmed that crowberry treatment significantly reduced the volume of transplanted tumor. IHC staining of tumor tissue showed that Ki67 and DEK were reduced. These effects point that $D E K$ is a key mediator of the crowberry-induced inhibition of CCA cell proliferation and metastasis in vitro and in vivo, and can be used as a target gene for CCA treatment.

EMT is a progressive biological phenomenon in which epithelial cells gradually obtain a mesenchymal cell phenotype, resulting in enhanced invasion and metastasis ${ }^{[36-37]}$. Wang et al. reported that COX-2 induces the loss of E-cadherin expression, resulting in the promotion of ovarian cancer cell invasiveness ${ }^{[38]}$. Due to tumor migration is associated with EMT process, we analyzed the expression of EMT-associated factors. After being treated with crowberry, our results demonstrated that, in CCA cells, the lateral and vertical migration were obviously decreased. In addition, epithelial marker E-cadherin was upregulated, after treatment with crowberry. In contrast, mesenchymal markers such as Vimentin and 
Snail were downregulated. Xu et al found that silencing $D E K$ inhibited cervical cancer tumorigenesis and metastasis by downregulating the Wnt/ $\beta$-catenin pathway ${ }^{[39]}$. Yang et al showed that after silencing the $D E K$ gene in lung cancer cells, the expression of E-cadherin was upregulated, and the levels of Vimentin and Snail were downregulated ${ }^{[40]}$. It was also found that $D E K$-dependent migration and EMT occurs via $\beta$ catenin/E-cadherin signaling in $\mathrm{HCC}$ cells ${ }^{[34]}$. In accordance with these results, we discovered that the silencing of $D E K$ prohibited EMT by reducing the expression of Vimentin and Snail, in contrast, promoting the expression of E-cadherin. When DEK was overexpressed, it can accelerate the EMT process.

The Akt/mTOR signaling pathway is an indispensable way that adjusts cell proliferative potential, migration, and invasion ${ }^{[41-42]}$. Scholars found that Akt/mTOR signaling pathway is activated in CCA, and the inhibition of this pathway can delay CCA progression ${ }^{[43-44]}$. Thus, we investigated the effect of crowberry on the Akt/mTOR signaling pathway. Specifically, in CCA cells the expression of p-Akt, p-S6, and p-4EBP1 was significantly downregulated. Report indicates that $D E K$ also stimulates Akt phosphorylation in myeloid-enriched progenitor cells ${ }^{[45]}$. The GeneMANIA database showed that there is an interaction between $D E K$ and Akt/mTOR signaling pathways. Therefore, we urgently sought to determine whether $D E K$ regulates the Akt/mTOR signaling pathway to inhibit CCA. Cui suggested that siDEK suppresses microglia-mediated neuronal cell death via the downregulation of the Akt pathway ${ }^{[46]}$. In this study, silencing the DEK gene significantly inhibited the expression of p-Akt, p-4EBP1, and p-S6, which is consistent with previous reports ${ }^{[47]}$, while the outcome of $D E K$ overexpression was opposite. Our previous results showed that the $D E K$ protein expression level was not changed when an Akt inhibitor was incubated with CCA cells, suggesting that $D E K$ is located upstream of the Akt signaling pathway ${ }^{[48]}$. However, the specific mechanism of whether DEK and Akt signaling have a direct or indirect action remains to be further studied. $D E K$ promotes cancer progression through many pathways. Yang indicated that $D E K$ promote the proliferation and invasion of breast cancer cells by activating the Wnt signaling pathway ${ }^{[12]}$. As mentioned above, the proliferation of CCA cells was inhibited after siDEK transfection. We speculated that the inhibition of CCA cell proliferation induced by DEK is achieved, at least in part, through the Akt signaling pathway. Whether other pathways are involved remains to be further studied. Phosphorylated and activated Akt can regulate the EMT by modulating the actin cytoskeleton in a cell[49] Song suggested that inhibition of DEK notably mitigated the bronchial EMT process in vitro and in vivo, and these effects may be interceded through the PI3K signaling pathway ${ }^{[50]}$. As we mentioned above, siDEK can inhibit the migration, EMT process and Akt signaling pathway in CCA cells, therefore, we have reason to believe that activation of the PI3K/AKT/mTOR signaling pathway is required for DEKstimulated CCA cell proliferation and migration.

\section{Conclusion}

In summary, we demonstrated that crowberry inhibited CCA cell proliferation and migration through a molecular mechanism that includes inhibition of $D E K$ and the Akt signaling pathway in vivo and vitro. Importantly, these data provide novel insights into the mechanisms of CCA and indicate that crowberry 
may be a novel agent for the development of therapies to improve CCA clinical treatment and prognosis. However, identifying the specific component of crowberry that is responsible for its antitumor effect still requires further research.

\section{Abbreviations}

Abbreviations: Akt, Serine/threonine Kinase; CCA, Cholangiocarcinoma; Cyt C, cytochrome C;

Caspase, Cysteinyl aspartate specific proteinase; EMT, epithelial-to-mesenchymal transition; EdU,

5-ethynyl-2'-deoxyuridine; HPLC, high-performance liquid chromatography; ig, intragastric

administration; MTT, 3-(4,5)-dimethylthiahiazo (-z-y1)-3,5-di- phenytetrazoliumromide; PARP, Poly

(ADP-ribose) polymerase; siRNA, small interfering RNA.

\section{Declarations}

\section{Availability of data and materials}

The datasets used and/or analyzed during the current study are available from the corresponding author upon reasonable request.

\section{Ethics approval and consent to participate}

All procedures were performed in accordance with the guidelines of the Yanbian University ethics committee.

\section{Consent for publication}

Not applicable.

\section{Conflict of interest}

The authors declare that there are no conflicts of interest.

\section{Acknowledgements}

We wish to thank all the staff members of the Key Laboratory of the Science and Technology Department of Jilin Province.

\section{Funding Sources}

This work was supported by National Natural Science Foundation of China (No.31460303, No.81660609, No.81860741), Jilin Provincial Science and Technology Department Project (No.20180101007JC) and 
Jilin Provincial Education Department Project (JJKH20191116KJ).

\section{Author Contributions}

\section{Affiliations}

Department of Pathology and Cancer Research Center, Yanbian University; Key Laboratory of Pathobiology (Yanbian University), State Ethnic Affairs Commission; Key Laboratory of the Science and Technology Department of Jilin Province; Research and Innovation Group of Yanbian University;

Xue Wang, Xin Zhang, Xuebing Zhou, Chunyu Yang, Yingshi Piao, Xiangshan Ren

Key Laboratory of Natural Medicines of the Changbai Mountain, Ministry of Education, Yanbian University;

Xue Wang,Xin Zhang, Xuebing Zhou, Chunyu Yang, Yingshi Piao, Xiangshan Ren, Ludan Zhang, Jinhua Zhao, Lili Jin, Renbo An

Department of Immunology and Pathogenic Biology, Yanbian University, Yanji 133002, China;

Guihua Jin

Co-author: Xue Wang, Ludan Zhang, Xin Zhang

\section{Contributions}

XS Ren and RB An provided financial support to conduct of the research and/or prepare the article. XW and XS Ren conceived and designed experiments. XW, LD Zhang and XZ performed the experiments. All authors provided support in the collection, analysis and interpretation of data. XW, XS Ren, RB An, GH Jin contributed to editing the manuscript and supervised the experimentators. All authors have read and agreed to submit the manuscript. All authors read and approved the final manuscript.

\section{Corresponding authors}

Correspondence to Xiangshan Ren or Renbo An/Guihua Jin.

\section{References}

[1] Doherty B, Nambudiri VE., Palmer WC. Update on the Diagnosis and Treatment of Cholangiocarcinoma. Curr Gastroenterol Rep. 2017; 19(1): 2. DOI: 10.1007/s11894-017-0542-4.

[2] Rizvi S, Khan SA, Hallemeier CL, Kelley RK, Gores GJ. Cholangiocarcinoma - evolving concepts and therapeutic strategies. Nat Rev Clin Oncol. 2018; 15(2): 95-111. DOI: 10.1038/nrclinonc.2017.157. 
[3] Koskela AK, Anttonen MJ, Soininen TH, Saviranta NM, Auriola S, Julkunen TR, Karjalainen RO. Variation in the anthocyanin concentration of wild populations of crowberries (Empetrum nigrum $L$ subsp. hermaphroditum). J Agric Food Chem. 2010; 58 (23): 12286-91. DOI: 10.1021/jf1037695.

[4] Park SY, Lee ES, Han SH, Lee HY, Lee S. Antioxidative effects of two native berry species, Empetrum nigrum var. japonicum K. Koch and Rubus buergeri Miq., from the Jeju Island of Korea. J Food Biochem. 2011; 36 (6):675-682. DOI: 10.1111/j.1745-4514.2011.00582.x.

[5] Claudia M, Fabíola C, Anna M P, Mysore VT, Martins MCL. Prevention of urinary catheter-associated infections by coating antimicrobial peptides from crowberry endophytes. Sci Rep. 2019; 9 (1):10753. DOI: 10.1038/s41598-019-47108-5.

[6] Kim KC, Kim D, Kim SC, Jung E, Park D, Hyun JW. Empetrum nigrum var. japonicum extract suppresses ultraviolet B-induced cell damage via absorption of radiation and inhibition of oxidative stress. Evid Based Complement Alternat Med. 2013; 983609. DOI: 10.1155/2013/983609.

[7] Ming Z, Shu LH, Dan W, Jun L, Jin LW, Li MB, Shun JZ. Chalcones from Empetrum nigrum var. Japonicum. Chinese Traditional and Herbal Drugs. 2016; 47 (24):4318-4321. DOI: 10.7501/j.issn.0253-2670.2016.24.003.

[8] Tunde JJM, Sona S, Stefan B, Jiri S, Mojmir B, Daniela S. Black Crowberry (Empetrum nigrum L.) Flavonoids and Their Health Promoting Activity. Molecules. 2016; 21 (12): 1685. DOI: $10.3390 /$ molecules 21121685 .

[9] León-González A J, López-Lázaro M, Espartero J L, Martín-Cordero C. Cytotoxic activity of dihydrochalcones isolated from Corema album leaves against HT-29 colon cancer cells. Nat Prod Commun. 2013; 8 (9):1255-1256. DOI: 10.1177/1934578X1300800918.

[10] Li CY. Study on the preventive effect of Northeast Yangaolan on alcoholic fatty liver in rats. Master Dissertation, Heilongjiang University of Chinese Medicine, China, 2009. http://kns.cnki.net/kns/detail/detail.aspx?FileName=2009175615.nh\&DbName=CMFD2009 (accessed 2021-10-08).

[11] Yong T, Li WL, Catherine EJ. The Complexity of DEK Signaling in Cancer Progression. Curr Cancer Drug Targets. 2018; 18 (3): 256-265. DOI: 10.2174/1568009617666170522094730.

[12] Mai QY, Lin LB, Zhao W, Lei, Yi WZ, Zhi HL, Wen JH, Chen CL, Hong TX. DEK is highly expressed in breast cancer and is associated with malignant phenotype and progression, Oncol Lett. 2021; 21 (6): 440. DOI: 10.3892/ol.2021.12701.

[13] Huo CY, Hong BD. Wen SS, Zheng JL, Ya LL. DEK overexpression is predictive of poor prognosis in esophageal squamous cell carcinoma. Arch Med Sci. 2019; 17 (1):135-141. DOI: 10.5114/aoms.2019.84210. 
[14] Ting Z, Bi JQ, Sen HZ, Guo PD, Li PC, Zheng RW. Expression of DEK in pancreatic cancer and its correlation with clinicopathological features and prognosis. J Cancer. 2019; 10 (4): 911-917. DOI: 10.7150/jca.27405.

[15] Soo YL, Wonkyung J, Jinhwan L, Aeree K, Han KK., Baek-Hui K. High expression of DEK is associated with poor prognosis in hepatocellular carcinoma. Histol Histopathol. 2019; 34 (11): 1279-1288. DOI: 10.14670/HH-18-125.

[16] Feng YT, Yu HW, Brian A H, Curzio R, Gong DX. PKB/Akt-dependent regulation of inflammation in cancer. Semin Cancer Biol. 2018; (48): 62-69. DOI: 10.1016/j.semcancer.2017.04.018.

[17] Michael R, Duggan Michael W, Kamel K. PAM (PIK3/AKT/mTOR) signaling in glia: potential contributions to brain tumors in aging. Aging (Albany NY). 2021; 13 (1): 1510-1527. DOI: 10.18632/aging.202459.

[18] Jiang M, Wu YL, Li X, Zhang YU, Xia KL, Cui BW, Li A, Li H, Nan JX. Oligomeric proanthocyanidin derived from grape seeds inhibited NFkB signaling in activated HSC: Involvement of JNK/ERK MAPK and PI3K/Akt pathways. Biomed Pharmacother. 2017; (93): 674-680. DOI: 10.1016/j.biopha.2017.06.105.

[19] Yang Y, Gao M, Lin Z, Chen L, Jin Y, Zhu G, Wang Y, Jin T. DEK promoted EMT and angiogenesis through regulating PI3K/AKT/mTOR pathway in triple-negative breast cancer. Oncotarget. 2017; 8 (58): 98708-98722. DOI: 10.18632/oncotarget.21864.

[20] Yan AZ, Jie L, Shi BW, Xiao LL, Yang L, Zhao HL, Jie Z, Jing L, Li HD, Qin Y. The DEK oncogene activates VEGF expression and promotes tumor angiogenesis and growth in HIF-1a-dependent and independent manners. Oncotarget. 2016; 7 (17): 23740-23756. DOI: 10.18632/oncotarget.8060.

[21] Diepenbruck M, Christofori G. Epithelial-mesenchymal transition (EMT) and metastasis: yes, no, maybe. Curr Opin Cell Biol. 2016; (43): 7-13. DOI: 10.1016/j.ceb.2016.06.002.

[22] Nye J, Melters DP, Dalal Y. The Art of War: harnessing the epigenome against cancer. F1000 Res. 2018; (7): 141. DOI: 10.12688/f1000research.12833.1.

[23] Wu X, Wu G, Wu Z, Yao X, Li G. MiR-200a Suppresses the Proliferation and Metastasis in Pancreatic Ductal Adenocarcinoma through Downregulation of DEK Gene. Trans Oncol. 2016; 9 (1):25-31. DOI: 10.1016/j.tranon.2015.11.005.

[24] Valle JW, Lamarca A, Goyal L, Barriuso J, Zhu AX. New horizons for precision medicine in biliary tract cancers. Cancer Discov. 2016; 7 (9): 943-962. DOI: 10.1158/2159-8290.CD-17-0245.

[25] Pedro MR, Paula O, Nuno AP, Irene O, Alona AL, Ana L, Luis B, Maria JP, Jesus MB. Pathogenesis of Cholangiocarcinoma. Annu Rev Pathol. 2021; (16)『433-463. DOI: 10.1146/annurev-pathol-030220020455区 
[26] Woo CC, Kumar AP, Sethi G, Tan KH. Thymoquinone:potential cure for inflammatory disorders and cancerl. Biochem Pharmacol. 2012; 83 (4):443-451. DOI: 10.1016/j.bcp.2011.09.029.

[27] Zhang Y, Chen J, Ma XM, Shi YP. Simultaneous determination of flavonoids in Ixeridium gracile by micellar electrokinetic chromatography. J Pharm and Biomed Anal. 2007; 45 (5): 742-

746. DOI: $10.1016 / j . j p b a .2007 .08 .014$.

[28] Sheng Y, Zou M, Wang Y, Li Q. 2',4'-dihydroxychalcone, a flavonoid isolated from Herba oxytropis, suppresses PC-3 human prostate cancer cell growth by induction of apoptosis. Oncol Lett. 2015; 10 (6): 3737-3741. DOI: 10.3892/ol.2015.3795.

[29] Pistritto G, Trisciuoglio D, Ceci C, Garufi A, D'Orazi G. Apoptosis as anticancer mechanism: function and dysfunction of its modulators and targeted therapeutic strategies. Aging (Albany NY). 2016; 8 (4):603-619. DOI: 10.18632/aging.100934.

[30] Hetz C, Vitte PA, Bombrun A, Rostovtseva TK, Montessuit S, Hiver A, Schwarz MK, Churc D J, Korsmeyer SJ, Martinou JC, Antonsson B. Bax channel inhibitors prevent mitochondrion- mediated apoptosis and protect neurons in a model of global brain ischemia. J Biol Chem. 2005; 280 (52):4296042970. DOI: 10.1074/jbc.M505843200.

[31] Slee EA, Adrain C, Martin SJ. Serial killers: ordering caspase activation events in apoptosis. Cell Death Differ.1999; 6 (11):1067-1074. DOI: 10.1038/sj.cdd.4400601.

[32] Wang YF, Chen C, Yang C, Li H, Yang YX. Effects of 6-Shogoal on the apoptosis of human colorectal cancer cells and the expression of bax, bcl2, caspase 3 and PARP1. Modern Food Technology. 2017; 33 (11): 7-15. DOI: 10.13982/j.mfst.1673-9078.2017.11.002.

[33] Riveiro FE, Soengas MS. Control of tumorigenesis and chemoresistance by the DEK oncogene. Clin Cancer Res. 2010;16 (11):2932-2938. DOI: 10.1158/1078-0432.CCR-09-2330.

[34] Yu L, Huang X, Zhang W, Zhao H, Wu G, Lv F, Shi L, Teng Y. Critical role of DEK and its regulation in tumorigenesis and metastasis of hepatocellular carcinoma. Oncotarget. 2016;7 (18):26844-26855.DOI: 10.18632/oncotarget.8565.

[35] Liu T, Zhu G, Yan W, Lv Y, Wang X, Jin G, Cui M, Lin Z, Ren X. Cordycepin Inhibits Cancer Cell Proliferation and Angiogenesis through a DEK Interaction via ERK Signaling in Cholangiocarcinoma. $J$ Pharmacol Exp Ther. 2020; 373 (2): 279-289, DOI: 10.1124/jpet.119.263202.

[36] Zhao M, Ang L, Huang J, Wang J. MicroRNAs regulate the epithelial-mesenchymal transition and influence breast cancer invasion and metastasis. Tumor Biol. 2017; 39 (2): 101042831769168. DOI: $10.1177 / 1010428317691682$.

[37] Sven J, Jamie A, Dominic DG, Kyra C, Geert B, Steven G. Epithelial-Mesenchymal Transition (EMT) as a Therapeutic Target. Cells Tissues Organs. 2021; (5):1-26. DOI: 10.1159/000512218. 
[38] Wang YP, Wang QY, Li CH, Li XW. COX-2 inhibition by celecoxib in epithelial ovarian cancer attenuates E-cadherin suppression through reduced Snail nuclear translocation. Chem Biol Interact. 2018; (292):2429. DOI: 10.1016/j.cbi.2018.06.020.

[39] Xu X, Zou L, Yao Q, Zhang Y, Gan L, Tang L.Silencing DEK downregulates cervical cancer

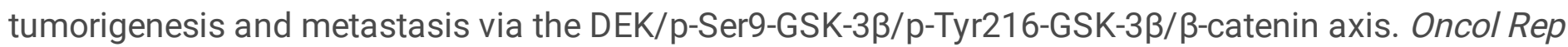
.2017; 38 (2): 1035-1042. DOI: 10.3892/or.2017.5721.

[40] Yang MQ, Bai LL, Lei L, Zheng YW, Wang Z, Li ZH, Liu CC, Huang WJ, Xu HT. DEK Promotes the Proliferation and Invasion of Lung Cancers and Indicates Poor Prognosis in Lung Adenocarcinomas. Oncol Rep. 2020; 43 (4):1338-1348. DOI: 10.3892/or.2020.7488.

[41] Chen J, Zhao KN, Li R, Shao RF, Chen C. Activation of PI3K/Akt/mTOR pathway and dual inhibitors of PI3K and mTOR in endometrial cancer. Curr Medl Chem. 2014; 21 (26): 3070-3080. DOI: $10.2174 / 0929867321666140414095605$.

[42] Hui H, Hong YZ, Jing ZC, Jiao W, Jie Y, Yang FJ. Targeting Akt in cancer for precision therapy. J Hematol Oncol. 2021; 14 (1):128. DOI: 10.1186/s13045-021-01137-8.

[43] Corti F, Nichetti F, Raimondi A, Niger M, Prinzi N, Torchio M, Tamborini E, Perrone F, Pruneri G, Di BM, de BF, Pusceddu S. Targeting the PI3K/AKT/mTOR pathway in biliary tract cancers: A review of current evidences and future perspectives. Cancer Treatment Reviews. 2019; (72): 45-55. DOI: 10.1016/j.ctrv.2018.11.001.

[44] Bishwanath A, Wanna C, Kesara NB. Atractylodin inhibited the migration and induced autophagy in cholangiocarcinoma cells via PI3K/AKT/mTOR and p38MAPK signalling pathways. J Pharm Pharmacol. 2021; 73 (9):1191-1200. DOI: 10.1093/jpp/rgab036.

[45] Maegan L, Capitano, Nirit MV, Anjan KS, Scott C, Maureen L, Haihong G, Rafael CG, Ferdinand K, Maureen AS, Christopher TL, Xin XH, David MM, Hal EB. Secreted nuclear protein DEK regulates hematopoiesis through CXCR2 signaling. J Clin Invest. 2019; 129 (6):2555-2570. DOI: $10.1172 / \mathrm{JCl} 127460$.

[46] Yan JC, Jee Y, Jin JW, Ji HL, Yoon SY, Moon SK, Sung CJ, Joo MP, Eun SY, Seong HK, Sangmee AJ, Kyoungho S, Su YE. Dieckol Attenuates Microglia-mediated Neuronal Cell Death via ERK, Akt and NADPH Oxidase-mediated Pathways. Korean J Physiol Pharmacol. 2015;19 (3):219-28.

DOI: 10.4196/kjpp.2015.19.3.219.

[47] Xue XC, Xian W, Wen O, Min G, Zi LG, Ming YS, Yun JC, Yan YL, Yong C, Hang X. Novel ent-Kaurane Diterpenoid From Rubus Corchorifolius L. F. Inhibits Human Colon Cancer Cell Growth via Inducing Cell Cycle Arrest and Apoptosis. J Agric Food Chem. 2017; 65 (8):1566-1573. D0I:10.1021/acs.jafc.6b05376 . 
[48] Te SL.The mechanisms of Cordycepin suppresses cell prohferatl07 $\mathrm{n}$ and migration by targeting DEK in cholangiocarcinoma via Akt and Erkl囚2 signaling pathway. Master Dissertation, Yanbian University, China, 2018. https://kns.cnki.net/kns8/defaultresult/index (accessed 2021-10-08).

[49] Mostafa KR, Arash S, Anvar S, Kolsoum RK, Amir RA, Mohammad S. Role of AKT and mTOR signaling pathways in the induction of epithelial-mesenchymal transition (EMT) process.

Biochimie. 2019; (165):229-234. DOI:10.1016/j.biochi.2019.08.003.

[50] Yi LS, Zhi GW, Jing ZJ, Yi HP, Li L, Chang X, Hong MP, Liang CL, Guang HY. DEK-targeting aptamer DTA-64 attenuates bronchial EMT-mediated airway remodelling by suppressing TGF- $\beta 1 /$ Smad, MAPK and PI3K signalling pathway in asthma. J Cell and Mol Med.2020;24 (23): 13739-13750. DOI: $10.1111 /$ jcmm. 15942.

\section{Figures}


Fig. 1

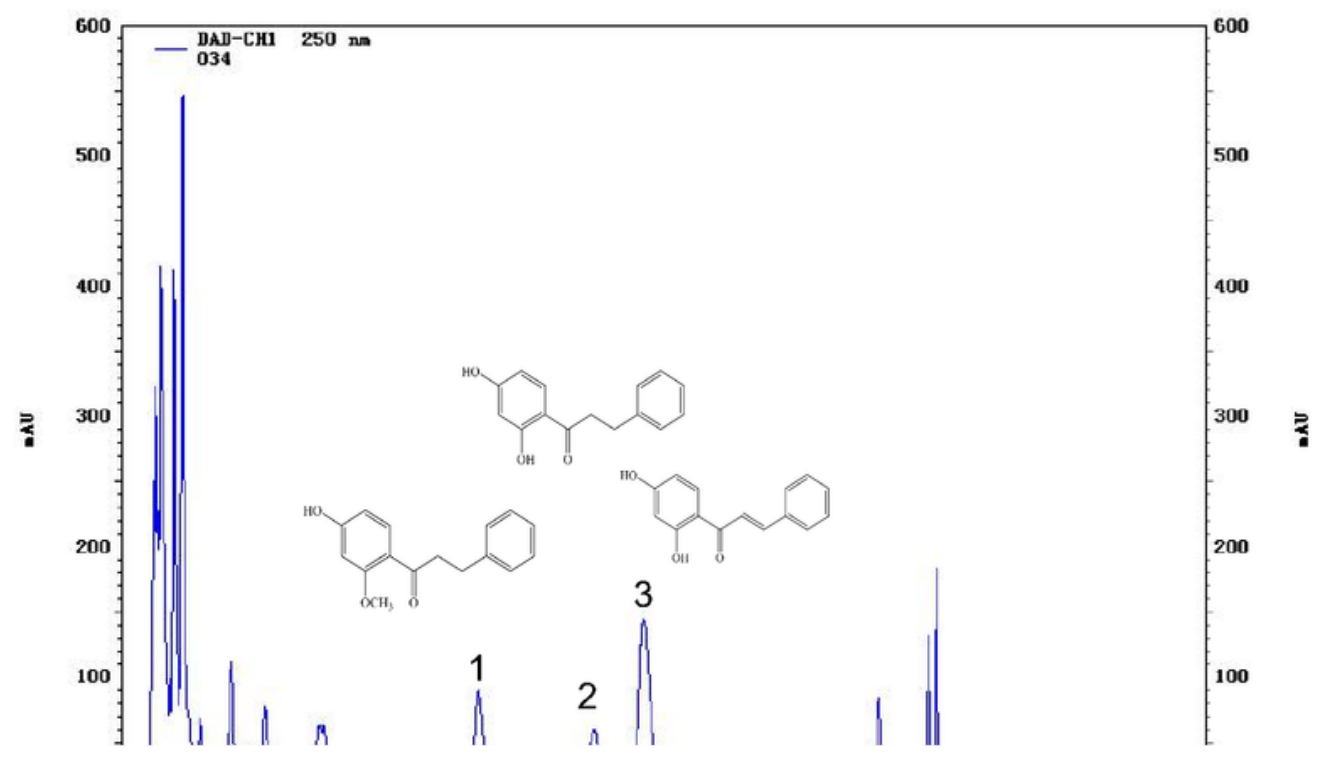

\section{Figure 1}

Bioactive compounds in crowberry extract

Structures of three flavonoid monomers and HPLC profiles of crowberry extract sample solution. 2'methoxy-4'-hydroxy-dihydrochalcone (1), 2',4'-dihydroxy-dihydrochalcone (2), and 2',4'-dihydroxy-chalcone (3) were determined at $250 \mathrm{~nm}$. 


\section{Figure 2}

\section{Crowberry suppresses the proliferation and colony formation of CCA cell lines}

MTT assay was used to detect the effect of crowberry on the viability in HuCCT1 and QBC939 cells (A). Colony formation assay to detect the ability of CCA cells (B). EdU assay to detect the proliferation of CCA cells after treatment with crowberry (C). ${ }^{\star} P<0.05,{ }^{\star} * P<0.01$ (vs CON group, $\mathrm{n}=3$ ); CON, control.

\section{Figure 3}

\section{Crowberry induces the apoptosis of CCA cells}

Hoechst33342 staining (white arrow) (A) and flow cytometry (B) were performed to detect the apoptosis of CCA cells. The expression of apoptosis marker proteins in CCA cells and statistical chart of western blotting assays (C). ${ }^{*} P<0.05,{ }^{\star} P<0.01$ (vs CON group, $\mathrm{n}=3$ ). Original magnifications, $\mathrm{x} 200$ (A). CON, control.

\section{Figure 4}

\section{Crowberry suppresses the migration and EMT of CCA cells}

Effect of crowberry on the horizontal distance of HuCCT1 and QBC939 cells (A). Vertical migration ability of cells (B). The expression of EMT marker proteins after treatment with crowberry and statistical chart of western blotting assays (C). ${ }^{\star} P<0.05,{ }^{\star} * P<0.01$ (vs CON group, $\mathrm{n}=3$ ). $\times 40(\mathrm{~A}), \times 200(\mathrm{~B})$. CON, control.

\section{Figure 5}

\section{Crowberry inhibits Akt/mTOR signaling pathway}

The key marker in Akt signaling pathway was tested by western blotting, after treatment with crowberry and the statistical chart of western blotting assays. ${ }^{*} P<0.05, * \star P<0.01$ (vs CON group, $\mathrm{n}=3$ ). CON, control.

\section{Figure 6}




\section{Crowberry suppresses cell proliferation and migration by regulating $D E K$}

The expression of DEK in HIBEpic, HuCCT1, QBC939, REE, TFK1 and after treatment with crowberry for 48 hours in HuCCT1 was dectected by western blotting. The statistical chart of western blotting assays is attaching ( $A$ and $B)$. The effect of gene silencing and overprseeion was presented by western blotting (C). After the $D E K$ gene was silenced and overpressed, the effects on cell proliferation, migration shown in $\mathrm{D}, \mathrm{E}$ and $\mathrm{F}$. ${ }^{\star} P<0.05,{ }^{*} P<0.01$ (vs $C O N$ in si $D E K$ group, vs Vector in overexpression group, $\mathrm{n}=3$ ). Original magnifications, $\times 40(\mathrm{~F})$. Original magnifications, $\times 200(\mathrm{G})$. CON, control. NC, negative control.

\section{Figure 7}

\section{Crowberry targets DEK and Akt/mTOR signaling pathway to inhibit EMT progression in CCA cells}

Results of p-Akt, Akt, p-S6, S6, p-4EBP1 and 4EBP1 were detected by silenced and overexpressed DEK in HuCCT cell (A); Expressions of E-cadherin, Vimentin, Snail were detected by silenced and overexpressed DEK in HuCCT cell (B). The statistical chart of western blotting assays is attaching. CON, control. NC, negative control. ${ }^{*} P<0.05,{ }^{*} P<0.01$ (vs CON in si $D E K$ group, vs Vector in overpression group, $\mathrm{n}=3$ )

\section{Figure 8}

\section{Crowberry inhibits CCA progression in vivo}

Body and tumor-bearing representative images of mice (A); Changes of mouse body weight over time after crowberry treatment(B); Changes in tumor volume over time after crowberry treatment(C); The effect of using crowberry on tumor weight(D); H\&E staining of liver to assess the toxicity of crowberry (E); The expression of $D E K$ and Ki67 in mouse tumor tissues illustrated by IHC staining(F-J). ** $P<0.01$ (vs CON group, $\mathrm{n}=5), \mathrm{CON}$, control. $\mathrm{ns}$, no significance.

\section{Supplementary Files}

This is a list of supplementary files associated with this preprint. Click to download.

- SupplementaryFigurePDF.pdf

- GraphicalAbstractPDF.pdf

- Highlights.docx 